\title{
A ligand-based 3D pharmacophore model for the $\mu$ opioid receptor: application to the morphinan class of opioids
}

\author{
Muhammad Faheem Asim, Gerhard Wolber, Mariana Spetea, Helmut Schmidhammer \\ From 16th Scientific Symposium of the Austrian Pharmacological Society (APHAR) \\ Vienna, Austria. 25-27 November 2010
}

\section{Background}

Opioid receptors belong to the rhodopsin subclass within the superfamily of $\mathrm{G}$ protein-coupled receptors (GPCR), which are characterized by the presence of seven transmembrane (7TM) helices. They interact with morphine and related opioid alkaloids as well as with endogenous opioid peptides. There are three main types of opioid receptors $(\mu, \delta, \kappa)$, which are differently implicated in opioid function. Ligands specifically targeting each opioid receptor type are of high interest both as research tools and potential therapeutic agents. Activation of the $\mu$ opioid receptor produces many other effects, besides its main involvement in pain control, including immunomodulation, respiratory depression, constipation, tolerance and physical dependence. With the lack of an experimental 3D structure of the $\mu$ opioid receptor, discovery of 3D pharmacophores for the receptor that can explain the activity of a series of ligands represents an important approach in drug discovery.

\section{Materials and methods}

A new ligand-based pharmacophore model for the $\mu$ opioid receptor was generated using the LigandScout program. A database consisting of morphinan derivatives was generated to provide the base for virtual screening and validation studies. Opioid receptor binding activity data earlier published by our group were included in this in-house opioid library and a general structure-activity relationship for morphinan compounds was established.

\section{Results}

A merged feature ligand-based pharmacophore model for the $\mu$ opioid receptor was generated using a highlyactive training set of morphinans (in-house opioid library). The model was optimized, validated and was shown to be able to identify highly active $\mu$ opioid ligands within a certain range, and excellent enrichments were achieved. The pharmacophore model indicated that the important features for the binding activity with the $\mu$ receptor are the presence of at least three hydrogen bond acceptors, one aromatic ring and one positive ionisable feature.

\section{Conclusions}

The availability of the present pharmacophore model is expected to provide a more rational hypothetical picture of the primary chemical features responsible for activity, to be a valuable tool for 3D virtual screening and thus to facilitate the design of novel active candidates targeting the $\mu$ opioid receptor.

\footnotetext{
Acknowledgements

Supported by the Austrian Research Fund FWF: P21350 and TRP 16-B18.

Published: 16 November 2010

doi:10.1186/1471-2210-10-S1-A4

Cite this article as: Asim et al:: A ligand-based 3D pharmacophore

model for the $\mu$ opioid receptor: application to the morphinan class of opioids. BMC Pharmacology 2010 10(Suppl 1):A4.
}

\footnotetext{
* Correspondence: helmut.schmidhammer@uibk.ac.at

Department of Pharmaceutical Chemistry, Institute of Pharmacy and Center for Molecular Biosciences, University of Innsbruck, 6020 Innsbruck, Austria
} 